\title{
High Frame Rate Synthetic Aperture 3D Vector Flow Imaging
}

Villagómez Hoyos, Carlos Armando; Holbek, Simon; Stuart, Matthias Bo; Jensen, Jørgen Arendt

\section{Published in:}

Proceedings of IEEE International Ultrasonics Symposium 2016

Link to article, DOI:

10.1109/ULTSYM.2016.7728664

Publication date:

2016

Document Version

Peer reviewed version

Link back to DTU Orbit

Citation (APA):

Villagómez Hoyos, C. A., Holbek, S., Stuart, M. B., \& Jensen, J. A. (2016). High Frame Rate Synthetic Aperture $3 D$ Vector Flow Imaging. In Proceedings of IEEE International Ultrasonics Symposium 2016 IEEE. https://doi.org/10.1109/ULTSYM.2016.7728664

\section{General rights}

Copyright and moral rights for the publications made accessible in the public portal are retained by the authors and/or other copyright owners and it is a condition of accessing publications that users recognise and abide by the legal requirements associated with these rights.

- Users may download and print one copy of any publication from the public portal for the purpose of private study or research.

- You may not further distribute the material or use it for any profit-making activity or commercial gain

- You may freely distribute the URL identifying the publication in the public portal 


\title{
High Frame Rate Synthetic Aperture 3D Vector Flow Imaging
}

\author{
Carlos A. Villagómez-Hoyos, Simon Holbek, Matthias Bo Stuart and Jørgen Arendt Jensen \\ Center for Fast Ultrasound Imaging, Department of Electrical Engineering, \\ Technical University of Denmark, DK-2800 Lyngby, Denmark
}

\begin{abstract}
D blood flow quantification with high spatial and temporal resolution would strongly benefit clinical research on cardiovascular pathologies. Ultrasonic velocity techniques are known for their ability to measure blood flow with high precision at high spatial and temporal resolution. However, current volumetric ultrasonic flow methods are limited to one velocity component or restricted to a reduced field of view (FOV), e.g. fixed imaging planes, in exchange for higher temporal resolutions. To solve these problems, a previously proposed accurate 2-D high frame rate vector flow imaging (VFI) technique is extended to estimate the 3-D velocity components inside a volume at high temporal resolutions $(<1 \mathrm{~ms})$. The full 3 -D vector velocities are obtained from beamformed volumetric data using synthetic aperture (SA) techniques combined with a 2-D matrix array. The method is validated using Field II simulations of flow along a straight vessel phantom and with complex flow from a 3-D computational fluid dynamics (CFD) model of a carotid bifurcation. Results from the simulations show that the 3-D velocity components are estimated with a mean relative bias of $-12.8 \%,-10 \%$ and $1.42 \%$ for the $V_{x}, V_{y}$ and $V_{z}$ respectively; each presented a mean relative standard deviation of $11.8 \%, 12.3 \%$ and $1.11 \%$.
\end{abstract}

\section{INTRODUCTION}

Quantifying blood flow is complex as it moves in all three spatial dimensions and furthermore varies as a function of time. However, such quantification is beneficial for the diagnosis of cardiovascular diseases. Therefore, research for methods that quantifies the full 3-D blood flow is an on-going effort in several medical imaging modalities.

In ultrasound, recent developments of novel imaging techniques, such as synthetic aperture [1] and plane wave [2] imaging, have allowed for the quantification of 2-D blood flow in an entire image plane at high temporal resolutions (< $1 \mathrm{~ms})$ [3], [4]. However, ultrasound imaging remains today mainly a 2-D imaging modality due to the technical complexity of manufacturing matrix probes for high-framerate 3-D acquisitions. Although the availability of these type of arrays remains limited, research into new cost-effective solutions for matrix probes is growing.

The first full 3-D ultrasonic flow measurements using a matrix array technology were presented by Pihl et al. [5], who extended the 2-D transverse oscillation (TO) technique [6] to a 3-D implementation operating on two imaging planes. Furthermore, Holbek et al. demonstrated the technique in-vivo [7] and increased the achievable frame rate to $2.1 \mathrm{kHz}$ [8]. However, these 3-D flow estimation techniques still operate on a line-by-line basis, which limits the velocity estimation to pre-determined imaging planes. In parallel, with a similar transducer, Provost et al. investigated the use of ultrafast techniques for estimating 1-D velocities in a volume, achieving high volume rates of around $2.3 \mathrm{kHz}$ [9].

In this work, a full 3-D velocity estimation method capable of achieving volume rates up to $2.25 \mathrm{kHz}$ is presented. The method is an extension from a high-frame-rate 2-D velocity estimation technique [10] to a 3-D implementation. The proposed method is based on synthetic aperture vector flow imaging (VFI) techniques [11] and inherits its advantage of high dynamic velocity ranges [12] due to the continuously available data. The velocity estimation is first briefly introduced in Section II, where the extension of the method from 2-D to 3-D is discussed. The method implementation details are then given in Section III. Results from the Field II simulations in both the straight vessel phantom and the computational fluid dynamics (CFD) model of a carotid bifurcation are found in Section IV.

\section{THEORY}

The 3-D vector velocity estimation is based on a previously published 2-D numerical triangulation algorithm [10]. The proposed method follows the same principles as its 2-D counterpart, where a triangulation procedure is applied to a grid of estimated directional velocities.

First, directional velocity estimates are obtained by a time domain cross-correlation of directional beamformed signals along two consecutive acquisitions, as described by Jensen [13], [14]. The directional velocities are estimated in a spherical grid along a set of beamformed angles $\left(\theta_{\text {line }}, \phi_{\text {line }}\right)$ centered at a point $\overrightarrow{r_{p}}$ to generate velocity curves $V\left(\theta_{\text {line }}, \phi_{\text {line }}\right)$. The beamformed angles are distributed to cover the full $360^{\circ}$ range in both elevation and azimuth.

The numerical triangulation is then performed by finding the intersection point between the estimated directional velocity curves from distinct transmit beams $k$. The intersection point between the estimated velocity curves is found using a minimum distance criterion (MDC) approach. Here, the angle estimation relies on estimating the difference between normalized velocities from distinct directional velocity curves. The use of a normalization factor is required to avoid that low velocity estimates are regarded as presenting smaller absolute differences. 
The selected angles $\left(\theta_{M D C}\right.$ and $\left.\phi_{M D C}\right)$ are the ones presenting the minimum difference and are estimated using:

$$
\underset{\left(\theta_{\text {line }}, \phi_{\text {line }}\right)}{\operatorname{Arg} \min } \sum_{k=1}^{M-1} \sum_{l=k+1}^{M}\left|\frac{V_{k}\left(\theta_{\text {line }}, \phi_{\text {line }}\right)-V_{l}\left(\theta_{\text {line }}, \phi_{\text {line }}\right)}{\min \left(V_{k}\left(\theta_{\text {line }}, \phi_{\text {line }}\right), V_{l}\left(\theta_{\text {line }}, \phi_{\text {line }}\right)\right)}\right|
$$

where $M$ is the number of transmit beams or emissions.

After the angles have been obtained, the velocity magnitude is estimated by performing directional beamforming along the estimated angles. The final velocity magnitude is obtained from the summation of beamformed lines from distinct transmit beams instead of the individual ones, effectively synthesizing the aperture [11].

\section{METHODS}

\section{A. Simulation setup}

A number of simulations were performed using Field II Pro [15]-[17], in which tissue and blood are modeled as a collection of random point scatterers. Approximately 10 point scatterers per resolution cell were sufficient to ensure a Gaussian distributed RF signal. The size of the resolution cell was calculated based on the receive F-number, transmit frequency, and pulse length. In each emission the scatterer position is updated. Two different types of phantoms are simulated; a straight vessel phantom with a parabolic flow, and a 3-D complex flow phantom from a carotid bifurcation CFD model [18].
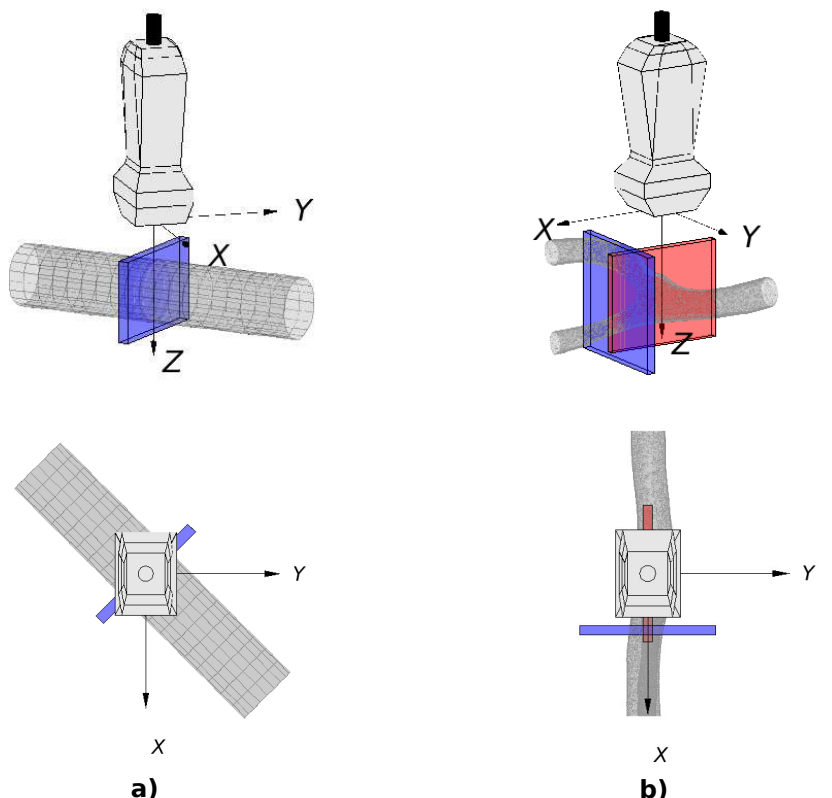

a)

b)

Fig. 1. Estimated planes for the simulated straight vessel (a) and the simulated CFD carotid bifurcation model (b).

1) Straight Vessel: Parabolic flow through a rigid vessel is simulated at flow angles of $\theta=90^{\circ}$ and $\phi=45^{\circ}$. The vessel has a radius of $6 \mathrm{~mm}$, centered at $15 \mathrm{~mm}$ depth, and with a non-moving vessel wall. The peak velocity in the vessel is
Table I

TRANSDUCER AND IMAGING PARAMETERS

\begin{tabular}{lllll}
\hline \multicolumn{2}{c}{ Transducer } & & \multicolumn{2}{c}{ Transmit Parameters } \\
\cline { 1 - 2 } \cline { 5 - 5 } Parameter & Value & & Parameter & Value \\
\hline Type & 2-D Matrix & & Excitation & 3 period sine \\
No. of elements & $32 \times 32$ & & Emitting elements & 1024 \\
pitch (both) & $0.3 \mathrm{~mm}$ & & Tx Apodization & 2-D Hanning \\
kerf (both) & $0.022 \mathrm{~mm}$ & & No. Tx Beams & 5 \\
$f_{0}$ & $3 \mathrm{MHz}$ & & PRF & $13.5 \mathrm{kHz}$ \\
& & & F-number & -3 \\
\hline
\end{tabular}

$0.5 \mathrm{~m} / \mathrm{s}$. Sixteen pulse emissions are used for calculating a velocity estimate, and fifty consecutive velocity estimations are performed for the statistics. The 3-D velocity components are estimated along the cross-sectional plane of the tube, as shown in Fig. 1. The estimated velocities are compared to the expected profile.

2) Carotid bifurcation: The carotid bifurcation model made publicly available by Swillens et al. is used in this investigation [18]. The model is a reconstruction from a CT-scan of a healthy volunteer, where an artificially eccentric plaque was added in the interna. The flow provided with the model was estimated using the CFD-package Fluent (ANSYS, Pennsylvania, USA) with an imposed inlet velocity profile obtained from an ultrasound velocity measurement of the same volunteer. Rigid walls are assumed in the CFD model, and no vessel wall or tissue movement was present. The cardiac cycle (length $1 \mathrm{~s}$ ) was divided into 200 equally spaced time steps in the CFD simulation.

The CFD velocity field information is coupled to positions of the point scatterer distribution used by Field II using a CFD-US simulation framework available at the authors' website (BioMMeda.ugent.be). The framework matches the large disparity in time scales between the CFD modeling $(5 \mathrm{~ms})$ and ultrasound simulations $(0.075 \mathrm{~ms})$ using an interframe linear interpolation. Additionally, a linear 3-D spatial interpolation from the CFD grid is made for each scatterer.

The 3-D velocity components are estimated along a longitudinal and a cross-sectional plane of the carotid bifurcation. The longitudinal plane is aligned to the center of the internal carotid, while the cross-sectional is taken at the output of the bifurcation, as shown in Fig. 1. Forty consecutive velocity estimates are calculated and the median is found.

\section{B. Imaging setup}

A 2-D 32x32 matrix array is simulated and RF data from 1024 channels are generated. A 5 emissions synthetic aperture flow sequence is used, with each emission consisting of an emulated spherical wave emanating from a virtual point source located behind the aperture. The virtual sources are angulated by $\pm 12^{\circ}$ with respect to the $\mathrm{z}$-axis against the other two axis, with the fifth emission coinciding with the $\mathrm{z}$-axis. The transmitted wavefront is, thus, directed towards a volume of interest (VOI), which is insonified in every emission (Fig. 2). The complete transducer and imaging parameters are listed in Table I. 

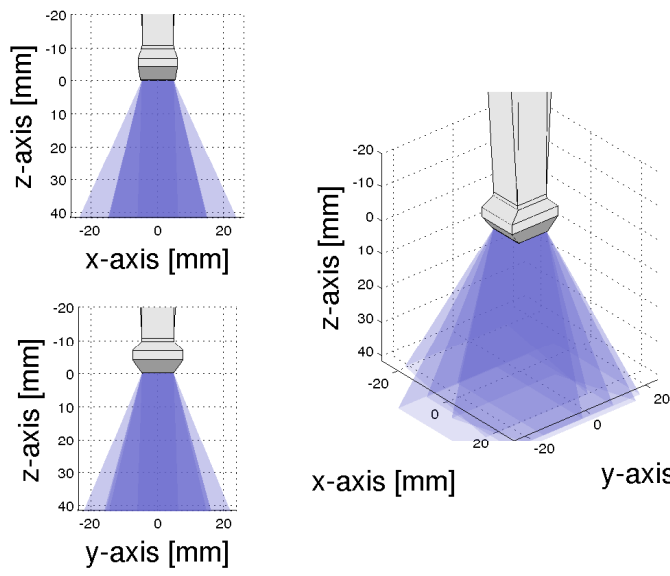

$\mathrm{X}$-axis $[\mathrm{mm}]$ $\mathrm{y}$-axis $[\mathrm{mm}$

Fig. 2. Overlay of the insonified area from each emission, along the $\mathrm{XZ}$ and YZ plane (left), and the full volume of interest shown in a darker shade (right).

\section{RESULTS}

1) Straight Vessel: The estimated 3-D velocities across a cross-sectional plane of the straight vessel phantom are shown in Fig. 3. The mean velocity magnitude is shown on top as a surface plot with casted shadows, both resembling the simulated parabolic profile. The mean velocity and standard deviations (SD) of estimated velocity components at the center line are shown below with the expected profile in red. The relative bias with respect to the true parabolic profile are $-12.8 \%,-10 \%$ and $1.42 \%$ for $\mathrm{V}_{x}, \mathrm{~V}_{y}$ and $\mathrm{V}_{z}$ respectively; each presented a mean relative standard deviation of $11.8 \%, 12.3 \%$ and $1.11 \%$.

2) Carotid bifurcation: Vector flow images (VFI) of the longitudinal and the cross-sectional planes are shown in Fig. 4 (a) and (b), respectively. Only the in-plane velocity components are considered in these plots. The reference velocities from the CFD model are shown on top, and the estimated velocity fields from ultrasound in the bottom. In all cases the reference CFD velocities were interpolated to the exact same locations as the ones estimated with ultrasound matching the same time instances for the involved frame.

In general, the estimated VFI frames in Fig. 4 (a) and (b) show good agreement with the reference CFD velocities. On the longitudinal plane it is visible that a vortex proximal to the internal carotid, seen as the top vessel, is well detected. However, the small vortex proximal to the external carotid is almost undistinguishable compared to the CFD reference. An underestimation of the velocity magnitude is also present in both branches. The underestimation is larger inside the interna, where biases up to $-60 \%$ are present. The cause of such underestimation is unknown and should be further investigated. On the cross-sectional plane, good agreement is also observed within the reference frame and the estimated frame. However, small vortex features are not present on the estimated velocities probably due to the limited ultrasound system resolution which in this case is of around $0.5 \mathrm{~mm}$ axially.

In Fig. 4 (c), the full 3-D vector velocity components of
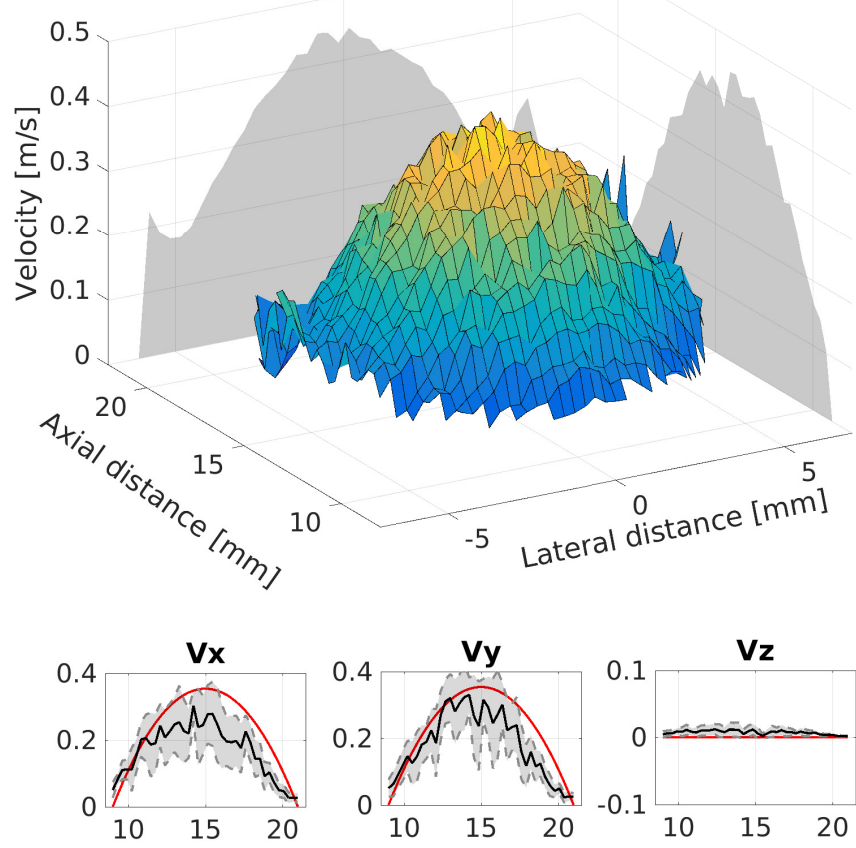

Fig. 3. Surface plot of the mean velocity magnitude across the cross-sectional plane of the straight vessel phantom (top). Mean and standard deviations (SD) of the estimated velocity components at the center line, with the true profile in red (bottom).

the same cross-sectional plane as (b) are shown. The velocity vectors are represented as arrows originating from the scan plane. The magnitude of velocity is encoded in the color on the arrow. The velocity underestimation is clearly visible in this figure.

\section{CONCLUSION}

The presented results showed that $3-\mathrm{D}$ vector velocities at a very high frame rate can be obtained in an entire volume. The simulations demonstrated that the angles can be estimated with sufficient accuracy in both simple and complex flow conditions. However, the computational cost of the method is high and further improvements need to be investigated. Additionally, validation of the method in experiments is needed before any clinical use. Nevertheless, the availability of complete 3-D components at high volumes rates offer a unique possibility to study complex flow patterns in 3-D. Furthermore, additional pathology indicators, such as pressure gradients, could be derived from the $3-\mathrm{D}$ vector flow.

\section{REFERENCES}

[1] M. Karaman, P. C. Li, and M. O’Donnell, "Synthetic aperture imaging for small scale systems," IEEE Trans. Ultrason., Ferroelec., Freq. Contr., vol. 42, pp. 429-442, 1995.

[2] L. Sandrin, S. Catheline, M. Tanter, X. Hennequin, and M. Fink, "Timeresolved pulsed elastography with ultrafast ultrasonic imaging," Ultrason. Imaging, vol. 21, no. 4, pp. 259-272, 1999. 

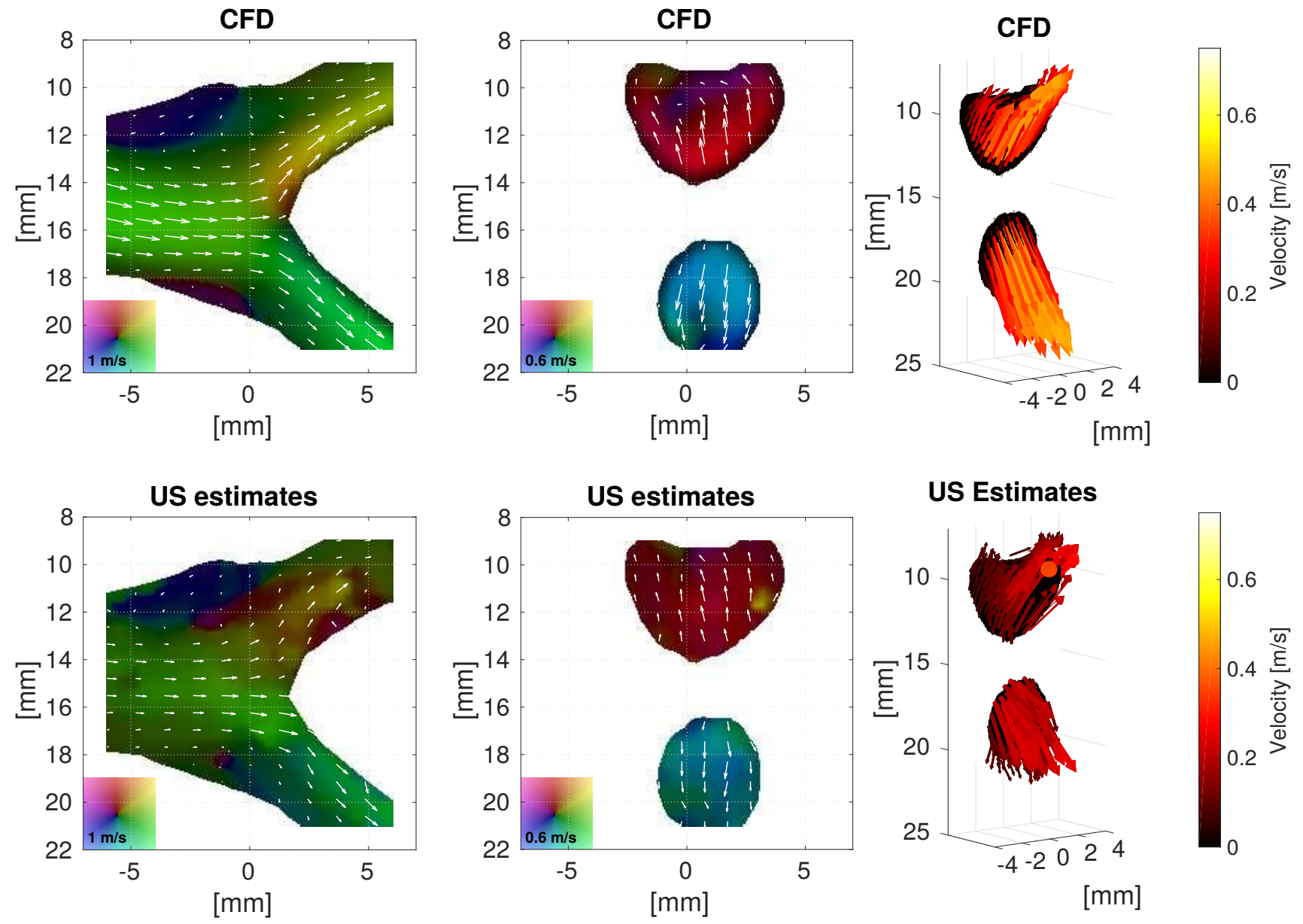

Fig. 4. Vector flow images (VFI) of the longitudinal and the cross-sectional planes, (a) and (b). A full 3-D vector representation of the cross-sectional plane is shown in (c). Reference velocities from the CFD are shown on top and estimated velocities are in the bottom. In the VFI frames the velocity magnitude and direction is represented according the the colormap in the left corner. In the full 3-D vector representation, the velocity magnitude is color encoded according to the colorbar.

[3] J. Udesen, F. Gran, K. L. Hansen, J. A. Jensen, C. Thomsen, and M. B. Nielsen, "High frame-rate blood vector velocity imaging using plane waves: simulations and preliminary experiments," IEEE Trans. Ultrason., Ferroelec., Freq. Contr., vol. 55, no. 8, pp. 1729-1743, 2008.

[4] B. Denarie, T. A. Tangen, I. K. Ekroll, N. Rolim, H. H. Torp, T. Bjastad, and L. Løvstakken, "Coherent plane wave compounding for very high frame rate ultrasonography of rapidly moving targets," IEEE Trans. Ultrason., Ferroelec., Freq. Contr., vol. 32, no. 7, pp. 1265-1276, 2013.

[5] M. J. Pihl and J. A. Jensen, "Measuring 3D velocity vectors using the transverse oscillation method," in Proc. IEEE Ultrason. Symp., 2012, pp. $1881-1885$.

[6] J. A. Jensen and P. Munk, "A new method for estimation of velocity vectors," IEEE Trans. Ultrason., Ferroelec., Freq. Contr., vol. 45, pp. 837-851, 1998.

[7] S. Holbek, M. Pihl, C. Ewertsen, M. Nielsen, and J. A. Jensen, "3-D velocity estimation for two planes in vivo," in Proc. IEEE Ultrason. Symp., 2014, pp. 1706-1709.

[8] S. Holbek, M. J. Pihl, C. Ewertsen, M. B. Nielsen, and J. A. Jensen, "In vivo 3-D vector velocity estimation with continuous data," in Proc. IEEE Ultrason. Symp., 2015, pp. 1-4.

[9] J. Provost, C. Papadacci, J. E. Arango, M. Imbault, M. Fink, J. L. Gennisson, M. Tanter, and M. Pernot, "3-D ultrafast ultrasound imaging in vivo," Phys. Med. Biol., vol. 59, no. 19, pp. L1-L13, 2014.

[10] C. A. Villagomez-Hoyos, M. B. Stuart, K. L. Hansen, M. B. Nielsen, and J. A. Jensen, "Accurate angle estimator for high frame rate 2-D vector flow imaging," IEEE Trans. Ultrason., Ferroelec., Freq. Contr., p. Accepted, 2016
[11] J. A. Jensen and S. I. Nikolov, "Directional synthetic aperture flow imaging," IEEE Trans. Ultrason., Ferroelec., Freq. Contr., vol. 51, pp. $1107-1118,2004$.

[12] C. A. Villagomez-Hoyos, M. B. Stuart, and J. A. Jensen, "In-vivo high dynamic range vector flow imaging," Proc. IEEE Ultrason. Symp., pp. $1-4,2015$

[13] J. A. Jensen, "Directional velocity estimation using focusing along the flow direction: I: Theory and simulation," IEEE Trans. Ultrason. Ferroelec., Freq. Contr., vol. 50, pp. 857-872, 2003.

[14] J. A. Jensen and S. I. Nikolov, "A method for real-time three-dimensional vector velocity imaging," in Proc. IEEE Ultrason. Symp., 2003, pp. 15821585.

[15] J. A. Jensen, "Field: A program for simulating ultrasound systems," Med. Biol. Eng. Comp., vol. 10th Nordic-Baltic Conference on Biomedical Imaging, Vol. 4, Supplement 1, Part 1, pp. 351-353, 1996.

[16] J. A. Jensen and N. B. Svendsen, "Calculation of pressure fields from arbitrarily shaped, apodized, and excited ultrasound transducers," IEEE Trans. Ultrason., Ferroelec., Freq. Contr., vol. 39, pp. 262-267, 1992.

[17] J. A. Jensen, "A multi-threaded version of Field II," in Proc. IEEE Ultrason. Symp. IEEE, 2014, pp. 2229-2232.

[18] A. Swillens, L. Løvstakken, J. Kips, H. Torp, and P. Segers, "Ultrasound simulation of complex flow velocity fields based on computational fluid dynamics," IEEE Trans. Ultrason., Ferroelec., Freq. Contr., vol. 56, no. 3 , pp. 546-556, 2009. 